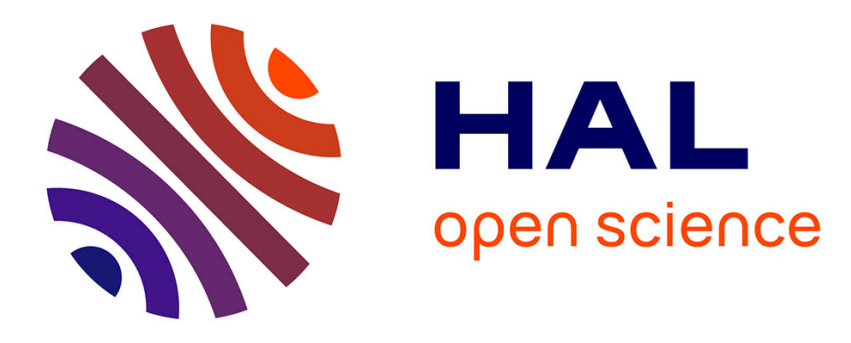

\title{
Yaëlle Amsellem-Mainguy, Arthur Vuattoux, Enquêter sur la jeunesse. Outils, pratiques d'enquête, analyses
}

\author{
Jeanne Demoulin
}

\section{To cite this version:}

Jeanne Demoulin. Yaëlle Amsellem-Mainguy, Arthur Vuattoux, Enquêter sur la jeunesse. Outils, pratiques d'enquête, analyses. Lectures, 2017, 10.4000/lectures.28557 . hal-03086273

\section{HAL Id: hal-03086273 \\ https://hal.parisnanterre.fr/hal-03086273}

Submitted on 6 Jan 2021

HAL is a multi-disciplinary open access archive for the deposit and dissemination of scientific research documents, whether they are published or not. The documents may come from teaching and research institutions in France or abroad, or from public or private research centers.
L'archive ouverte pluridisciplinaire HAL, est destinée au dépôt et à la diffusion de documents scientifiques de niveau recherche, publiés ou non, émanant des établissements d'enseignement et de recherche français ou étrangers, des laboratoires publics ou privés. 


\title{
Yaëlle Amsellem-Mainguy, Arthur Vuattoux, Enquêter sur la jeunesse. Outils, pratiques d'enquête, analyses
}

\author{
Jeanne Demoulin
}

1 Plutôt qu'un protocole clé en main, cet ouvrage se présente comme un manuel méthodologique pour enquêter sur la jeunesse. D'un côté, l'accent est mis sur les enjeux épistémologiques et les étapes de mise en œuvre de l'enquête. De l'autre, la mobilisation d'un riche corpus d'enquêtes consacrées à cet objet offre de fait au lecteur une série de contenus thématiques sur la sociologie de la jeunesse. Structuré en trois parties, l'ouvrage de Yaëlle Amsellem-Mainguy et Arthur Vuattoux suit les trois phases classiques d'une enquête : sa préparation, sa réalisation, puis sa restitution et le retour réflexif sur l'enquête. De plus, le découpage de chacune des parties en chapitres aux intitulés clairs et en lien direct avec les temps forts de la réalisation d'une enquête ${ }^{1}$ permet de naviguer facilement dans l'ouvrage et se prête aussi bien à une lecture extensive qu'à une lecture aléatoire.

2 Après avoir donné quelques éléments de contexte sur la jeunesse par le biais d'un rapide « portrait social » (p. 21) centré sur le processus d'autonomisation et les impacts de la crise économique sur la jeunesse, la première partie - intitulée «Préparer l'enquête " - répond à l'une des questions majeures qui vient à l'enquêteur souhaitant réaliser un travail empirique : où trouver les enquêtés? À cet égard, les indications fournies par les auteurs permettent d'une part d'identifier des espaces où rencontrer des jeunes, quel que soit le sujet travaillé - les institutions fréquentées par les jeunes, leurs familles, leurs groupes de pairs. D'autre part, des spécifications sur certains espaces sont données, en rapport avec des thématiques d'enquête telles qu'internet, la politique, ou encore les mouvements sociaux. Sur ces différents aspects, les auteurs apportent tant du contenu théorique que des pistes méthodologiques. Le chapitre sur les « groupes de pairs» rappelle ainsi les acquis de la recherche sur la "présence forte et importante des pairs dans le processus de construction identitaire» (p.51) et les enjeux scientifiques et méthodologiques qui en découlent. Il s'agit par exemple de 
«replacer les relations amicales dans la totalité du réseau relationnel des jeunes " (p. 53), de comprendre les termes qu'ils utilisent pour se définir (groupe, potes, bande, etc.) et de les interroger sur ces termes - tout en identifiant les personnes qu'ils évoquent pour aider les enquêtés à parler de cas concrets.

Dans cette première partie comme dans le reste de l'ouvrage, les auteurs ont à cœur de souligner la nécessité d'adopter une posture réflexive. À propos des institutions, ils rappellent par exemple qu'« en choisissant les institutions enquêtées, on choisit également d'enquêter sur une partie de l'espace social » (p. 35) qui révèle des choix en termes de classe et de sexe, de même que d'identification ethnique, nationale ou religieuse des enquêtés. Aussi est-il souligné que pour pouvoir être réalisée, une enquête qui passe par une institution devra être acceptée par cette institution. L'enjeu est alors pour l'enquêteur de prendre en compte non seulement «les contraintes des jeunes à participer » (p.36), dans la mesure où l'institution aura demandé à "ses » jeunes d'y prendre part; mais plus encore de " "sonder" le rapport qu'entretient l'institution qu'il veut étudier à la recherche» (p.37), notamment pour assurer la faisabilité de l'enquête et comprendre la liberté ou au contraire le contrôle exercé sur les enquêteurs. Si ces précautions sont d'usage pour toute enquête, qu'elle porte sur la jeunesse ou sur un autre objet, l'originalité du propos réside principalement dans les nombreux exemples apportés par les auteurs. Ainsi, un encadré retrace par exemple trois enquêtes réalisées dans différentes institutions connues des enquêtrices pour pointer les effets de ces institutions sur l'accès au terrain ${ }^{2}$.

4 La deuxième partie documente trois méthodes d'enquête classiques en sciences sociales - l'entretien, l'observation et le questionnaire - tout en donnant des éléments permettant de mettre en évidence la spécificité de leur utilisation quand elles sont appliquées à une enquête sur la jeunesse. Concernant l'entretien par exemple, les auteurs soulignent que les jeunes sont habitués à de multiples formes d'entretiens avec leur médecin, leurs professeurs, etc. L'un des enjeux est par conséquent $d^{\prime}$ '« expliquer clairement aux jeunes ce qui distingue l'entretien sociologique d'autres formes d'entretiens connues par les jeunes» (p. 81). Tout au long de cette partie, les auteurs insistent sur la spécificité des effets de distance ou de proximité sociale qui existent entre enquêteurs et enquêtés, notamment du fait de l'âge. Si les enquêteurs peuvent être assimilés à d'autres figures adultes connues des jeunes, telles que leurs enseignants ou leurs éducateurs par exemple (p. 100), ils doivent néanmoins veiller à ne pas se croire "supérieur[s] aux enquêtés » : cette situation, assez commune, peut ici se trouver « renforcée par l'illusion selon laquelle l'avancée en âge procurerait à celui qui enquête sur les jeunes une meilleure compréhension de la situation» (p. 106). En outre, les auteurs explorent aussi ce rapport aux jeunes au prisme de méthodes d'enquêtes moins répandues, comme les recherches qui «font avec » les jeunes en les impliquant dans l'enquête (chapitre 7). Toutefois, la documentation est globalement moins riche qu'ailleurs dans l'ouvrage, et les chapitres bien plus courts. Sans doute l'explication à cela est-elle relative au cadrage franco-français de l'ouvrage: les recherches participatives avec les jeunes ont par exemple fait l'objet d'une littérature anglophone abondante - notamment sous le vocable youth participatory action research et dont les résultats auraient pu venir utilement nourrir l'ouvrage.

5 La troisième partie - intitulée «Penser l'enquête, la restituer » - clôt l'ouvrage avec deux chapitres sur la posture réflexive de l'enquêteur et un dernier sur les enjeux de la restitution de l'enquête. Après de nombreuses allusions à l'importance de l'écart d'âge, 
cette partie finit par aborder plus frontalement la question: « Faut-il (et suffit-il d') être jeune pour enquêter sur les jeunes?» (titre du chapitre 9). Elle aborde également la problématique de la diversité des profils sociaux des jeunes et des rapports sociaux de genre, de classe et de race entretenus avec les enquêteurs ${ }^{3}$. Eu égard à l'enjeu qu'elle représente, la question des rapports sociaux entre enquêteurs et enquêtés aurait cependant davantage mérité de traverser l'ensemble de l'ouvrage. Au terme de ces deux chapitres, l'accent sera finalement mis au dernier chapitre sur les clés pour aborder la phase de restitution de l'enquête. Cette étape de restitution de l'enquête aux jeunes enquêtés peut par exemple être d'autant plus difficile que le temps de l'institution ne correspondra que rarement avec le temps de la recherche. Ainsi, le chercheur n'aura pas terminé sa recherche en l'espace d'une semaine de colonie de vacances... Selon les auteurs, il sera alors bienvenu de s'enquérir des coordonnées des enquêtés pour pouvoir leur transmettre des résultats. À cet égard, des précautions telles que l'adaptation du langage ou l'explication des principes d'une enquête sociologique seront bien sûr à privilégier, ceci afin qu'enquêteurs comme enquêtés puissent tirer profit des échanges effectués.

6 L'ouvrage pourra dès lors être utile à quiconque s'initie à la pratique de l'enquête, même si son objet n'est pas la jeunesse. Pour les enquêteurs et les chercheurs confirmés, l'intérêt résidera plutôt dans la mise en regard d'enjeux méthodologiques connus - car communs à de nombreux objets - avec une vaste palette d'exemples, quoiqu'issus quasi exclusivement d'une littérature francophone. L'originalité et les véritables apports de l'ouvrage se situent en effet au niveau de ses illustrations. Elles permettent au lecteur de voir plus concrètement quelles formes peuvent revêtir des enjeux méthodologiques classiques dans le contexte d'enquêtes portant sur la jeunesse. Si l'on peut regretter le cadrage strictement francophone des recherches mobilisées, ainsi qu'un traitement un peu rapide de la question des rapports sociaux entre enquêteurs et enquêtés, ce livre apparaît toutefois comme une synthèse réussie de multiples enjeux méthodologiques inhérents aux enquêtes en sciences sociales.

\section{NOTES}

1. À titre d'exemples, les chapitres 2 à 5 sont respectivement intitulés : « Entrer sur le terrain : où sont les jeunes? ", « Réaliser des entretiens avec des jeunes ", « Observation et immersion auprès des jeunes ", puis « Enquêter par questionnaire ».

2. Les cas évoqués correspondent aux environnements d'un «bon» lycée, de lycées professionnels et du milieu hospitalier (p. 38-39).

3. Voir le chapitre 10 : «Rapports de pouvoir et situations de violences au cours d'une enquête ».

\section{AUTHOR}

\section{JEANNE DEMOULIN}

Sociologue et urbaniste, docteure en études urbaines, chercheuse au LAVUE (UMR 7218 CNRS).

Recherche en cours : « les quartiers populaires au prisme de la jeunesse » (ANR Pop-Part). 